\title{
Modelling of the Deformation Diffusion Areas on a Para-Aramid Fabric with B-Spline Curves
}

\author{
Hatice Kuşak Samancı, ${ }^{1}$ Filiz Yağcı, ${ }^{2}$ and Ali Çalişkan ${ }^{3}$ \\ ${ }^{1}$ Department of Mathematics, Bitlis Eren University, Bitlis, Turkey \\ ${ }^{2}$ Department of Mathematics, Uludağ University, Bursa, Turkey \\ ${ }^{3}$ Department of Mathematics, Ege University, Izmir, Turkey \\ Correspondence should be addressed to Hatice Kuşak Samancı; ecitah_tamus@yahoo.com
}

Received 6 June 2016; Revised 29 August 2016; Accepted 15 September 2016; Published 20 February 2017

Academic Editor: Giuseppe Carbone

Copyright (C) 2017 Hatice Kuşak Samancı et al. This is an open access article distributed under the Creative Commons Attribution License, which permits unrestricted use, distribution, and reproduction in any medium, provided the original work is properly cited.

\begin{abstract}
The geometrical modelling of the planar energy diffusion behaviors of the deformations on a para-aramid fabric has been performed. In the application process of the study, in the experimental period, drop test with bullets of different weights has been applied. The B-spline curve-generating technique has been used in the study. This is an efficient method for geometrical modelling of the deformation diffusion areas formed after the drop test. Proper control points have been chosen to be able to draw the borders of the diffusion areas on the fabric which is deformed, and then the De Casteljau and De Boor algorithms have been used. The Holditch area calculation according to the beams taken at certain fixed lengths has been performed for the B-spline border curve obtained as a closed form. After the calculations, it has been determined that the diffusion area where the bullet with pointed end was dropped on a para-aramid fabric is bigger and the diffusion area where the bullet with rounded end was dropped is smaller when compared with the areas where other bullets with different ends were dropped.
\end{abstract}

\section{Introduction}

The exploration of the use of parametric curves and surfaces can be viewed as the origin of Computer Aided Geometric Design (CAGD). The major breakthroughs in CAGD were undoubtedly the theory of Bezier surfaces and Coons patches, later combined with B-spline methods. Bezier curves and surfaces were independently developed by P. De Casteljau at Citröen and by P. Bezier and Renault [1]. Gordon and Riesenfeld proposed curves and surfaces which use basis splines as blending functions. These are called B-spline curves and surfaces. B-spline curves, like Bezier curves, are defined by polygon vertices and have properties similar to those of Bezier curves. That is, B-spline curves are expressed as a convex combination of polygon vertex position vectors and also have the variation diminishing property. Curve shapes are smoothed versions of the polygon shapes and can be roughly predicted from the polygon shapes [2]. In plane geometry Holditch Theorem states if a chord of fixed length is allowed to rotate inside a convex closed curve then the locus of a point on the chord a distance $p$ from one end and a distance $q$ from the other is a closed curve whose area is less than that of the original curve by $\pi p q$. The theorem was published in 1858 by Rev. Amnet Holditch. While not mentioned by Holditch, the proof of the theorem requires an assumption that the chord be short enough that the traced locus is a simple closed curve [3].

In this research the Classic Holditch Theorem is given in [4]. Also in the paper [5] the authors used Holditch Theorem to calculate the areas between two quadratic uniform Bspline curves. In this study, the mathematical modelling of the deformation areas on para-aramid fabric has been investigated using the Holditch Theorem which calculated the areas between two uniform B-spline curves.

On the other hand, para-aramid fabrics produced from high-tenacity fibres are used in ballistic protection due to their high energy absorption ability and low tenacity/weight ratio. Ballistic behavior of textile fibres and fabrics has been investigated experimentally and ballistic behavior of textile fabric systems has been estimated in [6-15]. In our paper, 
TABLE 1: Properties of Twaron CT710 type fabrics used in this research, [7].

\begin{tabular}{lcccccc}
\hline Fabric Type & Count Warp/Weft & $\begin{array}{c}\text { Meterial Type } \\
\text { Warp/Weft }\end{array}$ & Weave & Density & Fabric Weight & Treatment \\
\hline CT 710 & $930 / 930$ & $2040 / 2000$ & Plain & $117 / 117$ & 220 & $\begin{array}{c}\text { Scouring/Water } \\
\text { repellent treatment }\end{array}$ \\
\hline
\end{tabular}

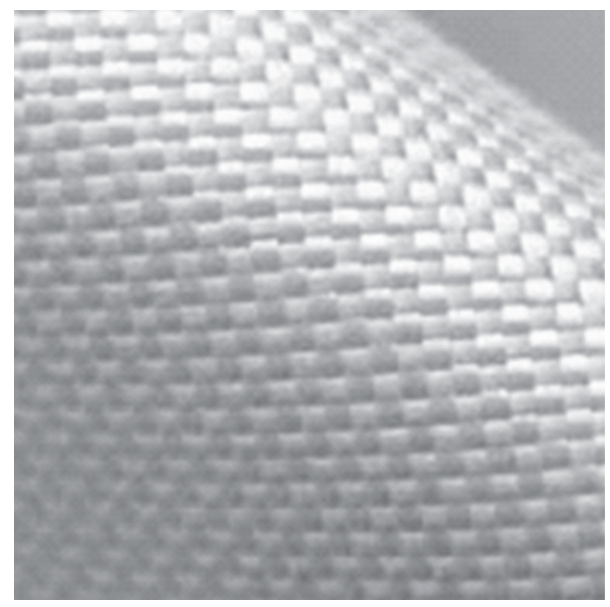

FIGURE 1: Structure of para-aramid fabric.

we have focused on a type of para-aramid fabric and we have investigated geometrical modelling of the planar energy diffusion behaviors of the deformations on the ballistic fabric.

\section{Material}

Para-aramid fabrics which are a class of heat-resistant and strong synthetic fabrics are used in aerospace and military applications for ballistic protection. In our research, Twaron CT type 710 fabric which is a type of para-aramid fabrics is used. Twaron fabric is one of ballistic fabrics used in steel vest production and developed in the early 1970s; see Figure 1.

With the help of textile engineers, the warp and wept information of the para-aramid fabric, which is in CT710 type, and other parameters of these fabrics as density and weight are shown in Table 1 . Fabrics were plied 1, 2, 4, 6, and 8 ply numbers and sewn $2,5 \mathrm{~cm}$ from edges with plain stich with high twist para-aramid sewing yarn. Fabric dimensions are $31 \times 31 \mathrm{~cm}$ and parameters of these fabrics are given in Table 1.

In our experiment step which was performed in the textile laboratory, the drop test was used under the $N_{i j}$ standards. The first material consists of three different bullets $45 \mathrm{~mm}$ in diameter, $1 \mathrm{~kg}$ in weight, consisting of a round tip shown with $A$, a middle butt-end tip shown with $B$, and a sharp tip shown with $\mathrm{C}$ in Figure 2.

The second material is the clay which has the characteristics of the human skin. Then the third material is the panel arm which is 1 meter in size and with which the bullet shots were made. The fourth material is the $50 \mathrm{~mm}$ pipe used for guiding the weight. Without using cloth for three bullet types and using 1 fold, 2 folds, 6 folds, and 8 folds, the number of the

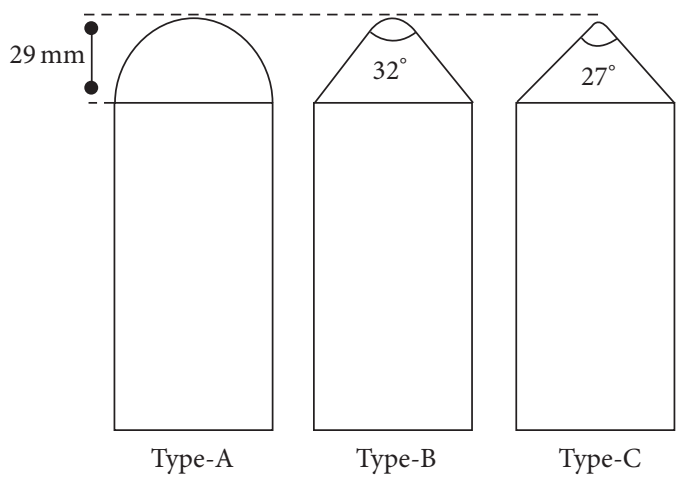

FIgURe 2: Bullets tip types.

shots was 72 , and 72 photos which were 2 -dimensional were taken in Figure 3.

The dropping tests are applied onto the fabric layers which are put on a clay base. The clay used is a special one which is in agreement with the characteristics of the human tissue. After the tests, the deformation on the clay is accepted as the possible deformation on the human tissue. The computation proceeded in four steps. Firstly, landmarks are identified from photographs and then landmark coordinates are determined. The coordinates of homologous landmarks are obtained from two shapes. These two shapes may represent either individual specimens or the means of two sets of shapes corresponding target landmarks. A transformation from a source shape to a target shape involves the displacement of the source landmarks to the corresponding target landmarks.

Note. Type Roma Plastilina (clay) which is given in $N_{i j}$ standards was used as clay material in this research. Clay was filled in a box as pointed out in the standard.

\section{Methods}

3.1. Classical Holditch Theorem. Let an $\mathrm{AB}$ chord with a constant length of $a+b$ on a circle (C) with a radius $r$ in Euclidean plane $E^{2}$ be divided by a point $D$ into two segments with lengths of $a$ and $b$, respectively. When the end points $\mathrm{A}$ and $\mathrm{B}$ of the chord draw the circle in full, then geometric location of $\mathrm{D}$ forms an inner circle. The difference between the areas of two closed curves can be calculated from the formula by $\pi \cdot a \cdot b$ as following Figure 4 in [4].

3.2. Using B-Splines for Geometric Modelling. In the early 1960s, J. C. Ferguson from the Boeing Airplane Company in the USA developed a method which defined the curves as vectors. A Ferguson Curve is a cubic vector function obtained with the parameters after determining the starting and ending 

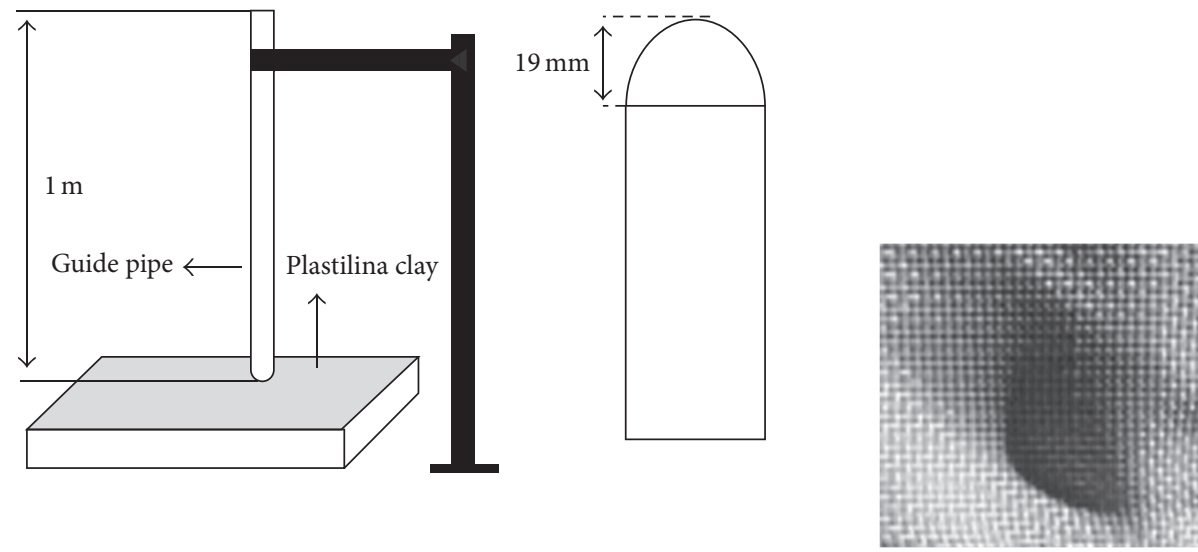

Figure 3: Test apparatus used in drop tests $[7,8]$.

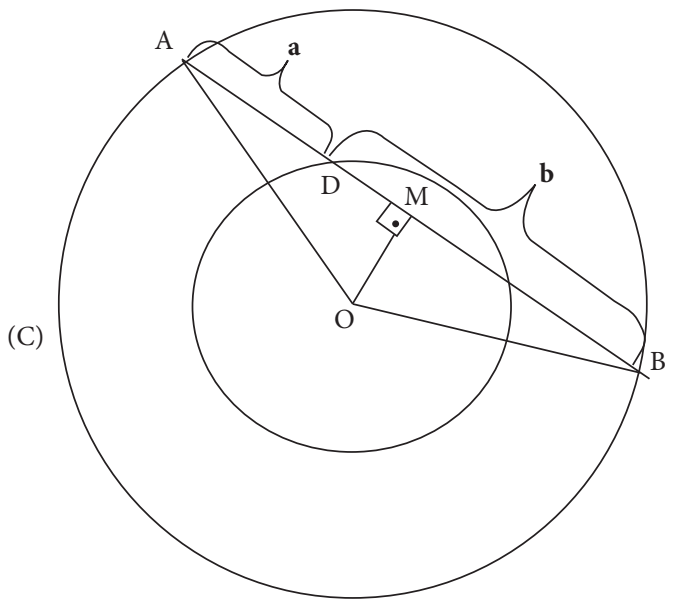

Figure 4: Classical Holditch Theorem [4].

points of a curve. Later in 1964, S. A. Coons introduced a surface definition method which gave the mathematical definition that provided the border conditions. In this method, the border curves and the position vectors in the 4 corners of the surface patch were taken into consideration. In 1974, P. Bezier from the Renault Company in France defined a curve representation by giving a polygon and called it as the Bezier curve. The Bezier curves were applied in practice to Renault automobile body designs. Gordon and Riesenfeld introduced the curves that used the base splines as combined functions and these curves were called B-spline curves. Like the Bezier curves, the B-spline curves are also defined by polygon corners and have similar properties. However, the Bspline curves differ from the Bezier curves in these points: The control points of the Bezier curves ensure only global control; however, the control points of the B-spline curves ensure local control. In other words, if a point is moved, the whole of the curve changes in the Bezier curve, but in the Bspline curve, only some parts are affected, and the other parts remain unchanged. For this reason, because of their ease in practice, the B-spline curves will be used in our study because they ensure local changes and the other parts of the curve do not change.

\subsection{De Casteljau Algorithm}

Given: $b_{0}, b_{1}, \ldots, b_{n} \in E^{3}$ and $t \in I R$

Set: $b_{i}^{r}(t)=(1-t) b_{i}^{r-1}(t)+t b_{i+1}^{r-1}(t)$, where: $r=$ $1, \ldots, n, i=0, \ldots, n-r$

and $b_{i}^{0}(t)=b_{i}$. Then $b_{0}^{n}(t)$ is the point with parameter value $t$ on the Bezier curve $b^{n}$.

The polygon $P$ formed by $b_{0}, b_{1}, \ldots, b_{n}$ is called the Bezier polygon or control polygon of the curve $b^{n}$. Similarly the polygon vertices $b_{i}$ are called control points or Bezier points. Sometimes we also write $b^{n}(t)=B\left[b_{0}, \ldots, b_{n} ; t\right]=B[P, t]$ or, shorter, $b^{n}=B\left[b_{0}, \ldots, b_{n}\right]=B P$. This notation defines $B$ to be the linear operator that associates the Bezier curve with its control polygon. We say that the curve $B\left[b_{0}, \ldots, b_{n}\right]$ is the Bernstein-Bezier approximation to the control polygon, a terminology borrowed from approximation theory. In the Bezier curves, the De Casteljau algorithm is used, because it ensures that the control points are taken in a certain order.

3.4. De Boor Algorithm. The De Boor algorithm is used in the design of the B-spline curves, because it ensures a certain method in taking the control points. Let $u \in\left[u_{1}, u_{I+1}\right) \subset$ $\left[u_{n-1}, u_{L+n-1}\right]$. Define

$$
\begin{array}{r}
d_{i}^{k}(u)=\frac{u_{i+n-k}-u}{u_{i+n-k}-u_{i-1}} d_{i-1}^{k-1}(u)+\frac{u-u_{i-1}}{u_{i+n-k}-u_{i-1}} d_{i}^{k-1}(u) \\
\text { for } k=1, \ldots, n-r
\end{array}
$$

and $i=I-n+k+1, \ldots, I-r+1$. Then $s(u)=\left[B_{n} P\right](u)=$ $d_{I-r+1}^{n-r}(u)$ is the value of the B-spline curve at parameter value $u$. Here, $r$ denotes the multiplicity of $u$ if it was already one of the knots. If it was not, set $r=0$. As usual, we set $d_{i}^{0}(u)=d_{i}$, $[1,2]$.

3.5. Uniform Closed B-Spline. A class $C^{M-2}$ closed curve (of degree $M-1)$ consisting of $n+1$ curve segments $P_{i}(t)$, 


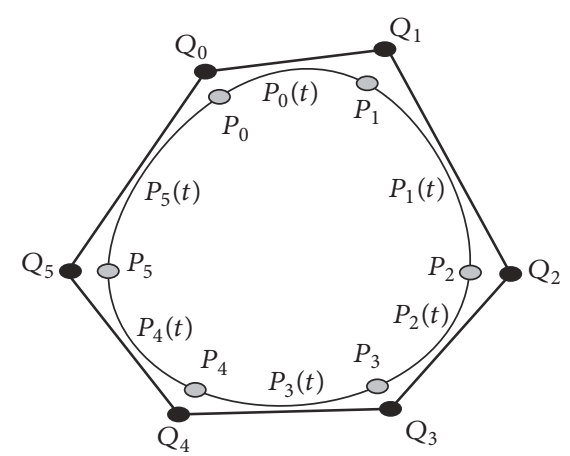

FIgURE 5: Uniform closed B-spline.

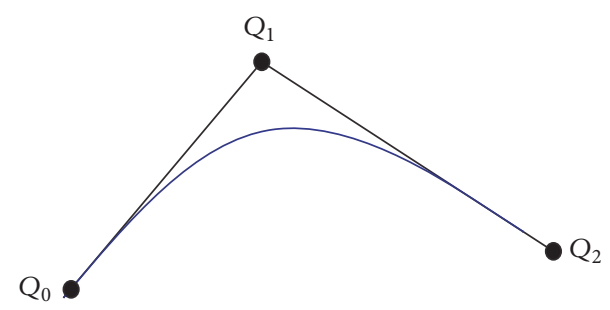

FIGURE 6: Quadratic B-spline segments.

$i=0,1, \ldots, n$, with a curve defining polygon determined by the $n+1$ position vectors $Q_{0}, Q_{1}, \ldots, Q_{n}$ in Figure 5 can be expressed by the following function:

$$
P_{i}(t)=\sum_{j=i-1}^{i+M+2} N_{j-i+1, M}(t) Q_{j \bmod (n+1)}, \quad 0 \leq t \leq 1,
$$

where the initial and finish points

$$
P_{i}(0)=P_{i}, \quad i=0,1, \ldots, n,
$$

are equal to each other, in $[1,2]$. We planned to use the uniform closed B-spline curves in our study because the photos of the deformation traces were taken in $2 \mathrm{D}$ during the experiment.

3.6. Location of Joints Uniform Quadratic B-Spline Segments. In [5], specifically each part is expressed as parabolas consisting of control points with three points because local control is ensured in B-spline curves in Figure 6. The matrix formats of these quadratic B-spline curves are as shown in the following equation:

$$
P_{i}(t)=\frac{1}{2} \cdot\left[\begin{array}{lll}
t^{2} & t & 1
\end{array}\right] \cdot\left[\begin{array}{ccc}
1 & -2 & 1 \\
-2 & 2 & 0 \\
1 & 1 & 0
\end{array}\right] \cdot\left[\begin{array}{c}
Q_{i-1} \\
Q_{i} \\
Q_{i+1}
\end{array}\right]
$$

In this case, only 3 control vertices are used in Figure 7. By joining these curves which correspond to these matrices, the closed curve is formed. In this situation, the midpoints of the two control points from the starting point are considered as being like tangents to the curves. Since the joining places

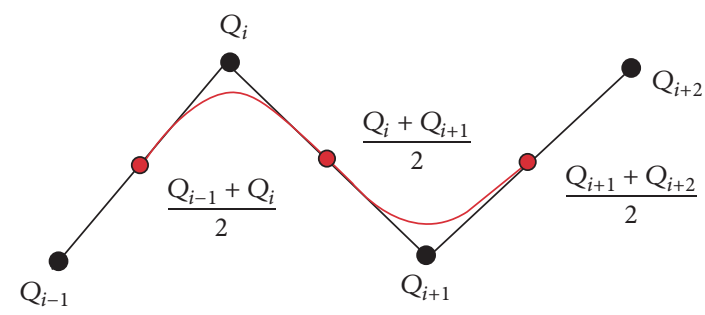

FIGURE 7: Location of joints uniform quadratic B-spline segments.

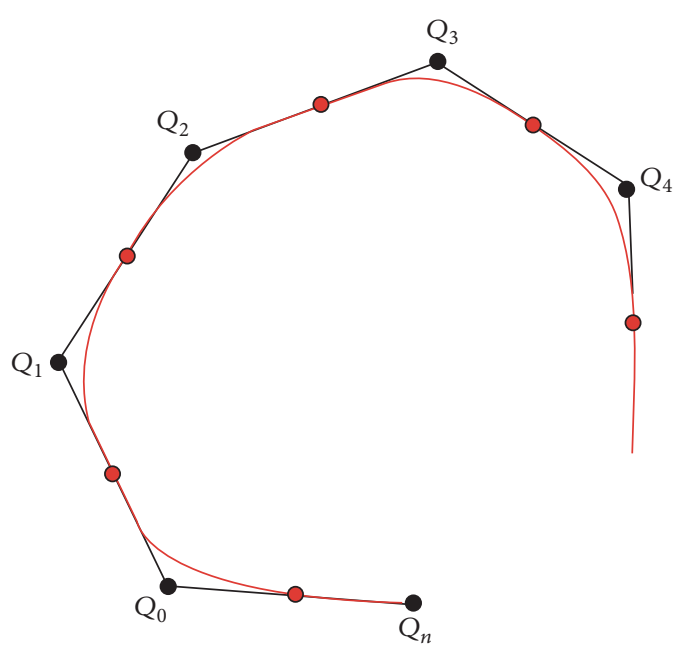

FIgURE 8: The curves with the C1 Continuity.

of these curves are the tangents, the curves are connected to each other continuously with the $\mathrm{C} 1$ Continuity in Figure 8.

When we expand this method by taking the $n$ point, the matrices in the following formulas are formed for each part:

$$
\begin{aligned}
& P_{0}(t)=\frac{1}{2} \cdot\left[\begin{array}{lll}
t^{2} & t & 1
\end{array}\right] \cdot\left[\begin{array}{ccc}
1 & -2 & 1 \\
-2 & 2 & 0 \\
1 & 1 & 0
\end{array}\right] \cdot\left[\begin{array}{l}
Q_{0} \\
Q_{1} \\
Q_{2}
\end{array}\right], \\
& P_{1}(t)=\frac{1}{2} \cdot\left[\begin{array}{lll}
t^{2} & t & 1
\end{array}\right] \cdot\left[\begin{array}{ccc}
1 & -2 & 1 \\
-2 & 2 & 0 \\
1 & 1 & 0
\end{array}\right] \cdot\left[\begin{array}{l}
Q_{1} \\
Q_{2} \\
Q_{3}
\end{array}\right], \\
& P_{n}(t)=\frac{1}{2} \cdot\left[\begin{array}{lll}
t^{2} & t & 1
\end{array}\right] \cdot\left[\begin{array}{ccc}
1 & -2 & 1 \\
-2 & 2 & 0 \\
1 & 1 & 0
\end{array}\right] \cdot\left[\begin{array}{l}
Q_{n} \\
Q_{0} \\
Q_{1}
\end{array}\right], \\
& (\beta):\left(P_{1}\right) \cup\left(P_{2}\right) \cup \cdots \cup\left(P_{n}\right)=\beta(t) .
\end{aligned}
$$

Uniform B-splines are convenient to represent closed curves. The only thing needed is a change in the number of the curve segments. Figure 9 shows a closed quadratic B-spline curve produced by 6 control points. Also we can find location of joints between uniform quadratic B-spline segments. If we take the vertices of this polygon as control points, quadratic uniform B-spline curve will pass from midpoints of sides. 


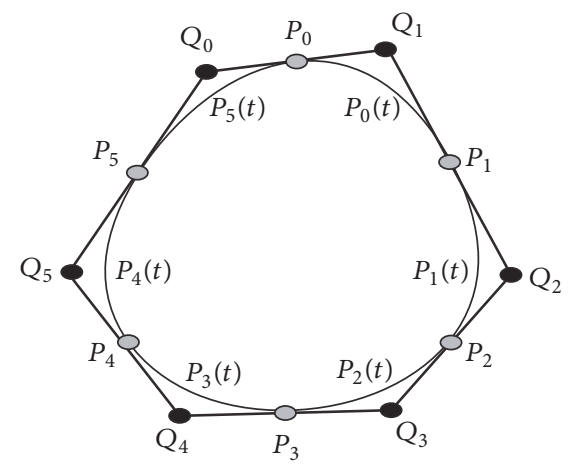

FIGURE 9: Different way of modelling uniform closed B-spline [5]

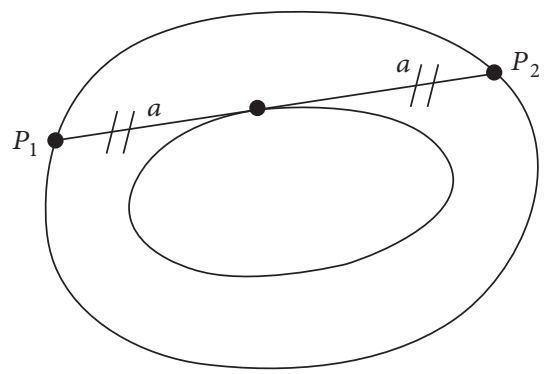

FIgure 10: Holditch Theorem with B-spline curves [5].

Equations of the curve segments will give us a closed curve for $n$ control points.

3.7. The Holditch Theorem by Using B-Splines. The $a$ and $b$ chords taken in the Classic Holditch Theorem are taken especially in equal lengths in $[3,4]$. We can find the area between the B-spline closed curves as

$$
\text { Area }=\pi \cdot a^{2}
$$

by the help of Holditch Classical Theorem in Figure 10.

\section{Main Results}

We applied these methods to the deformation photos at the end of the experiment. Our purpose is to calculate the area between the projection of the bullet and its effective area border curve with the help of the Holditch Theorem after drawing the B-spline curves. By doing so and by observing the deformation area in the cloth, we aim to determine which weight-end was used; see Figures 11 and 12.

4.1. Modelling of Deformation Splines for Ball Tip. The coordinates of big polygon for the ball tip in Figure 13 are given in the following:

$\begin{array}{ccccccc} & Q_{0}^{L} & Q_{1}^{L} & Q_{2}^{L} & Q_{3}^{L} & Q_{4}^{L} & Q_{5}^{L} \\ X & -12,7 & 12,15 & 15,21 & 5,04 & -12,09 & -15,62 \\ Y & 12,11 & 23,35 & -4,55 & -15,19 & -11,56 & 2,79 \\ & P_{0}^{L} & P_{1}^{L} & P_{2}^{L} & P_{3}^{L} & P_{4}^{L} & P_{5}^{L} \\ X & -0,275 & 13,68 & 10,125 & -3,525 & -13,855 & -14,16 \\ Y & 17,73 & 9,4 & -9,87 & -13,375 & -4,385 & 7,45\end{array}$

Spline Interpolation of Big Polygon for Ball Tip

$$
\begin{aligned}
P_{0}^{L} & =\left(-10.835 t^{2}+24.85 t-0.275,-19.57 t^{2}+11.24 t\right. \\
& +17.73), \\
P_{1}^{L} & =\left(-6.615 t^{2}+3.06 t+13.68,8.63 t^{2}-27.9 t+9.4\right), \\
P_{2}^{L} & =\left(-3.48 t^{2}-10.17 t+10.126,7.135 t^{2}-10.64 t\right. \\
& -9.87), \\
P_{3}^{L} & =\left(6.8 t^{2}-17.13 t-3.525,5.36 t^{2}+3.63 t-13.375\right), \\
P_{4}^{L} & =\left(3.225 t^{2}-3.53 t-13.855,-2.515 t^{2}+14.35 t\right. \\
& -4.385) .
\end{aligned}
$$

Small Polygon for the Ball Tip

$$
\begin{array}{ccccccc} 
& Q_{0}^{S} & Q_{1}^{S} & Q_{2}^{S} & Q_{3}^{S} & Q_{4}^{S} & Q_{5}^{S} \\
X & -3,62 & -0,09 & 3,54 & 3,54 & 0,01 & -3,66 \\
Y & 2,22 & 4,25 & 2,08 & -2,29 & -4,32 & -2,15 \\
& P_{0}^{S} & P_{1}^{S} & P_{2}^{S} & P_{3}^{S} & P_{4}^{S} & P_{5}^{S} \\
X & -1,855 & 1,725 & 3,54 & 1,775 & -1,825 & -3,64 \\
Y & 3,235 & 3,165 & -0,105 & -3,305 & -3,235 & 0,035
\end{array}
$$

Spline Interpolation of Small Polygon for Ball Tip

$$
\begin{aligned}
& P_{0}^{S}=\left(0.05 t^{2}+3.53 t-1.855,-2.1 t^{2}+2.03 t+3.235\right), \\
& P_{1}^{S} \\
& \quad=\left(-1.81 t^{2}+3.63 t+1.725,-1.1 t^{2}-2.17 t+3.165\right), \\
& P_{2}^{S}=\left(-1.765 t^{2}+3.54,1.17 t^{2}-4.37 t-0.105\right), \\
& P_{3}^{S}=\left(-0.07 t^{2}-3.53 t+1.775,2.1 t^{2}-2.03 t-3.305\right), \\
& P_{4}^{S}=\left(1.855 t^{2}-3.67 t-1.825,1.1 t^{2}+2.17 t-3.235\right) .
\end{aligned}
$$

\subsection{Modelling of Deformation Splines for Sharp Tip}

Big Polygon for the Sharp Tip in Figure 14

$$
\begin{array}{ccccccc} 
& Q_{0}^{L} & Q_{1}^{L} & Q_{2}^{L} & Q_{3}^{L} & Q_{4}^{L} & Q_{5}^{L} \\
X & -9,79 & -1,09 & 10,19 & 10,55 & 1,97 & -11,71 \\
Y & 3,91 & 12,47 & 4,13 & -3,62 & -11,12 & -3,86 \\
& P_{0}^{L} & P_{1}^{L} & P_{2}^{L} & P_{3}^{L} & P_{4}^{L} & P_{5}^{L} \\
X & -5,44 & 4,55 & 10,37 & 6,26 & -4,87 & -10,75 \\
Y & 8,19 & 8,3 & 0,255 & -7,37 & -7,49 & 0,025
\end{array}
$$

Spline Interpolation of Big Polygon for Sharp Tip

$$
\begin{aligned}
& P_{0}^{L}=\left(1.29 t^{2}+8.7 t-5.44,-8.45 t^{2}+8.56 t+8.19\right), \\
& P_{1}^{L}=\left(-5.46 t^{2}+11.28 t+4.55,0.295 t^{2}-8.34 t+8.3\right),
\end{aligned}
$$



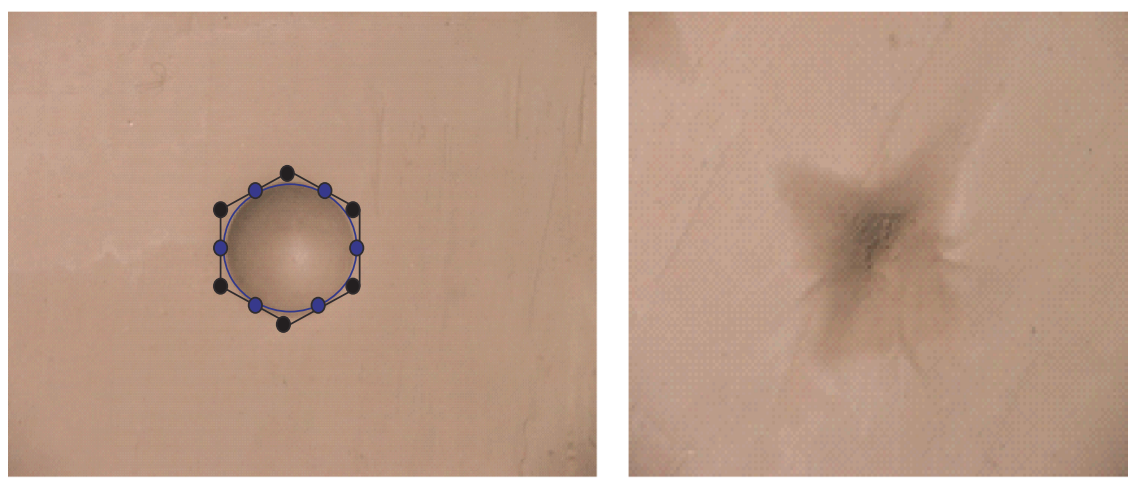

FIGURE 11: Deformation area with ball type.
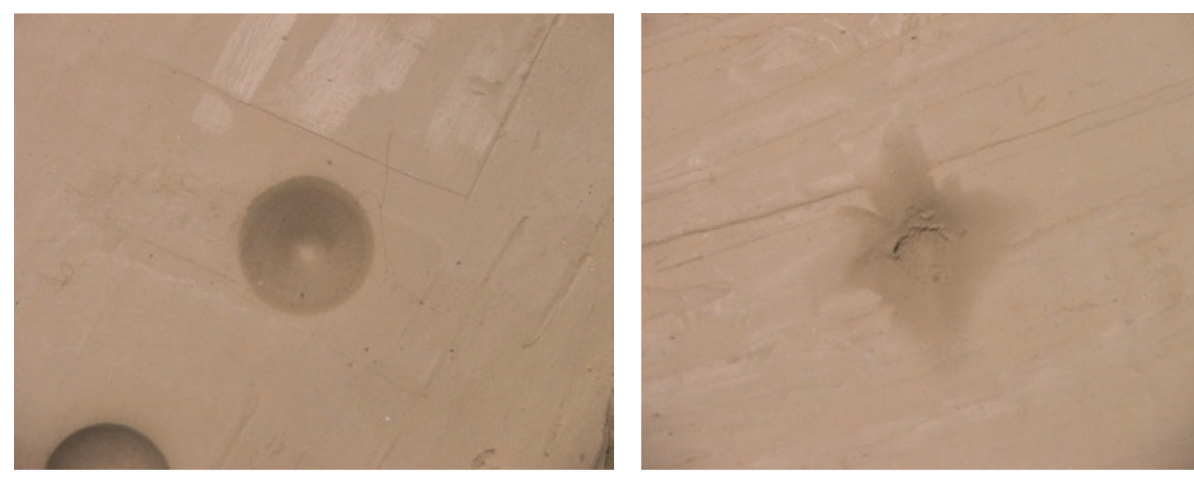

FIGURE 12: Deformation areas with sharp type.
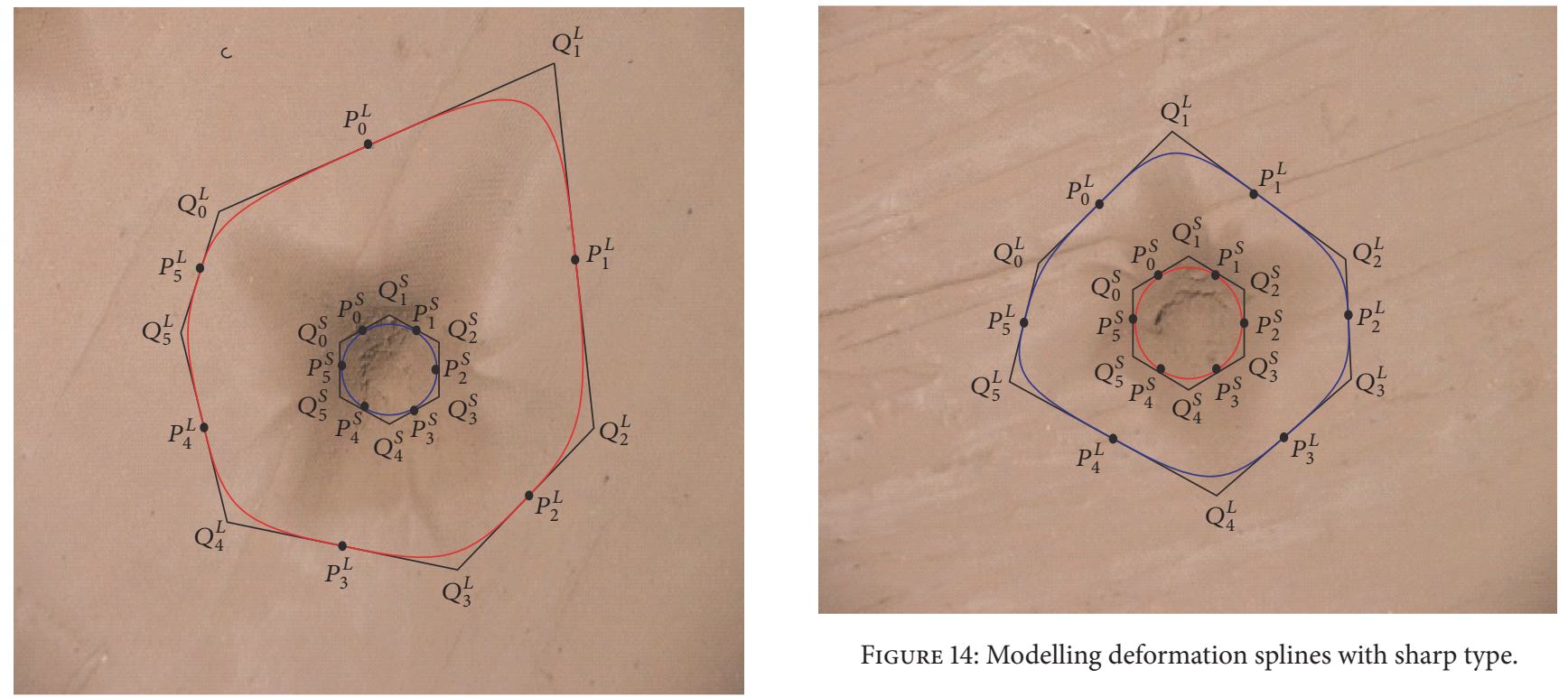

FIGURE 14: Modelling deformation splines with sharp type.

FIGURE 13: Modelling deformation splines with ball type.

$$
\begin{aligned}
& P_{3}^{L}=\left(-2.55 t^{2}-8.58 t+6.26,7.38 t^{2}-7.5 t-7.37\right), \\
& P_{4}^{L}=\left(7.80 t^{2}-13.68 t-4.87,0.255 t^{2}+7.26 t-7.49\right) .
\end{aligned}
$$

$=\left(-4.47 t^{2}+0.36 t+10.37,0.125 t^{2}-7.75 t+0.255\right)$, 


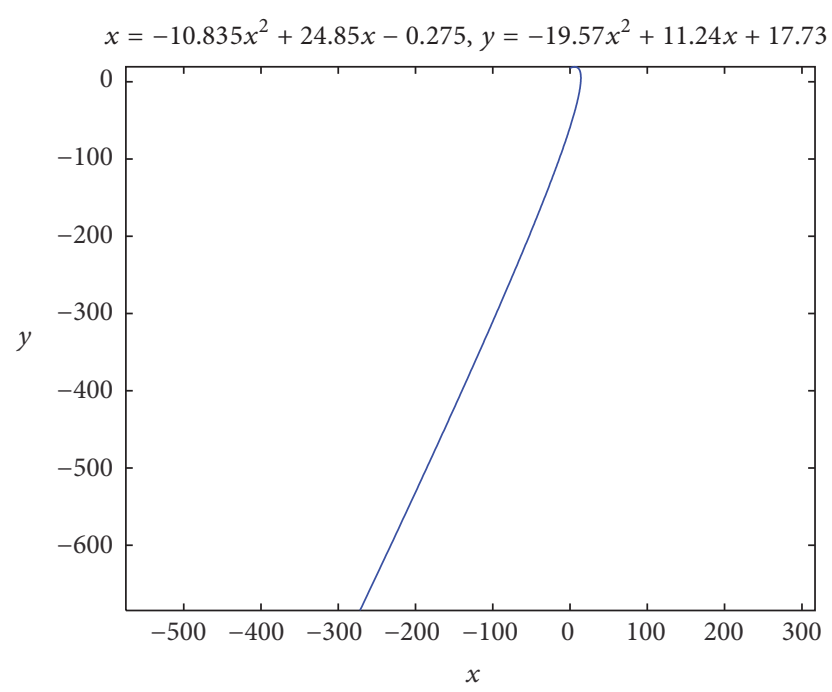

Figure 15: An example of geometric modelling by MATLAB.

Small Polygon for the Sharp Tip

$$
\begin{array}{ccccccc} 
& Q_{0}^{S} & Q_{1}^{S} & Q_{2}^{S} & Q_{3}^{S} & Q_{4}^{S} & Q_{5}^{S} \\
X & -3,59 & 0 & 3,66 & 3,66 & 0,06 & -3,59 \\
Y & 2,19 & 4,37 & 2,17 & -2,17 & -4,36 & -2,18 \\
& P_{0}^{S} & P_{1}^{S} & P_{2}^{S} & P_{3}^{S} & P_{4}^{S} & P_{5}^{S} \\
X & -1,795 & 1,83 & 3,66 & 1,86 & -1,765 & -3,59 \\
Y & 3,28 & 3,27 & 0 & -3,265 & -3,27 & 0,005
\end{array}
$$

Spline Interpolation of Small Polygon for Sharp Tip

$$
\begin{aligned}
& P_{0}^{S} \\
& \quad=\left(0.035 t^{2}+3.59 t-1.795,-2.19 t^{2}+2.18 t+3.28\right), \\
& P_{1}^{S}=\left(-1.83 t^{2}+3.66 t+1.83,-1.07 t^{2}-2.2 t+3.27\right), \\
& P_{2}^{S}=\left(-1.8 t^{2}+3.66,1.075 t^{2}-4.34 t\right), \\
& P_{3}^{S} \\
& \quad=\left(-0.025 t^{2}-3.6 t+1.86,2.185 t^{2}-2.19 t-3.265\right), \\
& P_{4}^{S} \\
& \quad=\left(1.825 t^{2}-3.65 t-1.765,1.095 t^{2}+2.18 t-3.27\right) .
\end{aligned}
$$

These splines can be modelling in the MATLAB program; see Figure 15. For example, the spline interpolation of ball tip

$$
\begin{aligned}
P_{0}^{L} & =\left(-10.835 t^{2}+24.85 t-0.275,-19.57 t^{2}+11.24 t\right. \\
& +17.73)
\end{aligned}
$$

can be represented in the MATLAB program as shown in Figure 15.

Also, the area between the B-spline closed curves can be found by using (6); thus Table 2 shows the areas for all tips.
TABLE 2: Calculating the expansion areas of tips.

\begin{tabular}{lc}
\hline & 8 Ply of fabric \\
\hline Type-A, ball type & 971.6465 \\
Type-B, blunt type & 598.7112 \\
Type-C, sharp type & 529.1348 \\
\hline
\end{tabular}

\section{Conclusion}

In our paper, the diffusion areas were modelled by a geometrical spline method. Some special points around the expansion area were taken on the deformation photos. Then the geometric spline modelling was done. As was mentioned above, the data of the Holditch areas that were found by using spline method after the geometric modelling study for each tip are given. The geometric spline method is very useful for the estimation of the expansion areas of tips. When we consider the tables, in estimating the bullet tip by observing the spread area, we can say that the spread area that is left by the bullet with round tip is more than the area which is left by the bullet with sharp tip. In our research we can see that using the Holditch area theorem with geometric spline method is very useful method in determining the tips from deformation photographs.

\section{Competing Interests}

The authors declare that they have no competing interests.

\section{Acknowledgments}

This study has received support from the Uludağ University Scientific Research Unit with the Project Reference no. UAP(F)-2012/21. The authors would like to thank Professor Ali Çalışkan for his valuable comments and suggestions in improving the quality of the paper.

\section{References}

[1] G. Farin, Curves and Surfaces for Computer Aided Geometric Design, A Practical Guide, Academic Press, 2nd edition, 1990.

[2] F. Yamaguchi, Curves and Surfaces in Computer Aided Geometric Design, Springer, Berlin, Germany, 1988.

[3] http://en.wikipedia.org/wiki/Holditch\%27s_theorem.

[4] K. Yucekaya Gülay and H. H. Hacisalihoğlu, Holditch's Theorem for Circles in 2-Dimensional Euclidean Space, vol. 18, Dumlupınar Universitesi Fen Bilimleri Dergisi, April 2009.

[5] A. Çalışkan and B. Tantay, "Application of Holditch theorem to bezier and quadratic uniform B-Spline curves," Mathematical and Computational Applications, vol. 6, no. 3, pp. 169-176, 2001.

[6] M. Karahan, A. Kuş, and R. Eren, "An investigation into ballistic performance and energy absorption capabilities of woven aramid fabrics," International Journal of Impact Engineering, vol. 35, no. 6, pp. 499-510, 2008.

[7] M. Karahan, F. Gülsoy, and S. Gündoğan, "The determination of energy propagating behaviour of woven para-aramid fabrics by $2-\mathrm{D}$ thin plate spline method," in Proceedings of the SAMPE Symposium Proceeding CD, Baltimore, Md, USA, June 2007. 
[8] F. Gulsoy, H. Kuşak Samancı, and A. Çalışkan, "Investigation of the bending energy and the expansion areas of the energy on Kflex fabrics," in Proceedings of the 14th World Textile Conference (AUTEX '14), 2014.

[9] M. Karahan, "The effect of fibre volume fraction on damage initiation and propagation of woven carbon-epoxy multi-layer composites," Textile Research Journal, vol. 82, no. 1, pp. 45-61, 2012.

[10] M. Karahan, "Comparison of ballistic performance and energy absorption capabilities of woven and unidirectional aramid fabrics," Textile Research Journal, vol. 78, no. 8, pp. 718-730, 2008.

[11] M. Fahool and A. R. Sabet, "Parametric study of energy absorption mechanism in Twaron fabric impregnated with a shear thickening fluid," International Journal of Impact Engineering, vol. 90, pp. 61-71, 2016.

[12] D. Dimeski, V. Srebrenkoska, and N. Mirceska, "Ballistic impact resistance mechanism of woven fabrics and their composites," Journal of Engineering Research \& Technology, vol. 4, no. 12, pp. 107-111, 2015.

[13] V. P. W. Shim, V. B. C. Tan, and T. E. Tay, "Modelling deformation and damage characteristics of woven fabric under small projectile impact," International Journal of Impact Engineering, vol. 16, no. 4, pp. 585-605, 1995.

[14] A. Tabiei and G. Nilakantan, "Ballistic impact of dry woven fabric composites: a review," Applied Mechanics Reviews, vol. 61, no. 1, Article ID 010801, 13 pages, 2008.

[15] P. M. Cunniff, "An analysis of the system effects in woven fabrics under ballistic impact," Textile Research Journal, vol. 62, no. 9, pp. 495-509, 1992. 


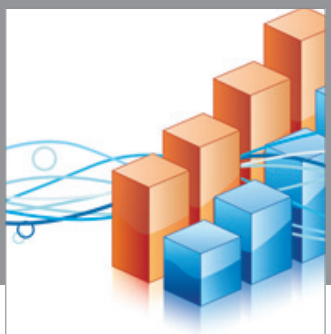

Advances in

Operations Research

vatem alat4

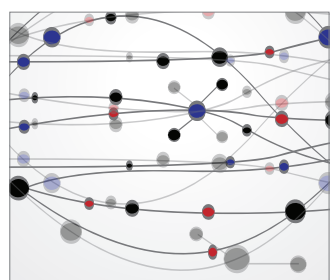

\section{The Scientific} World Journal
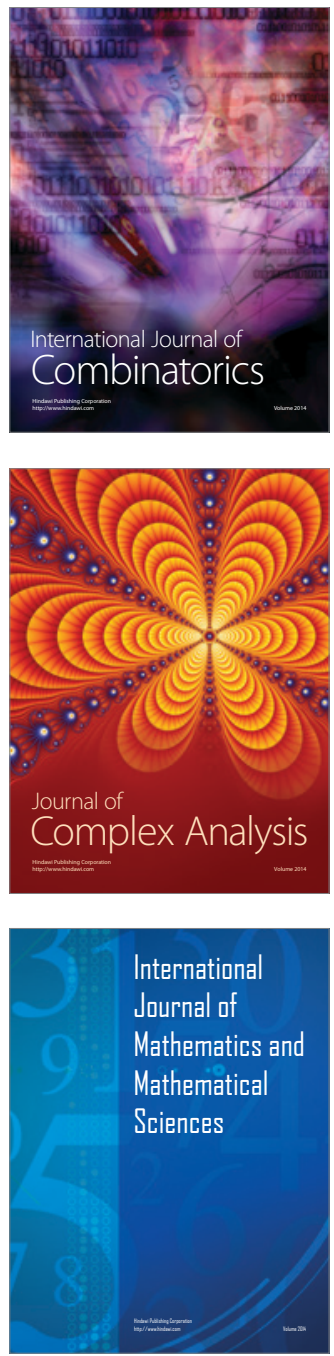
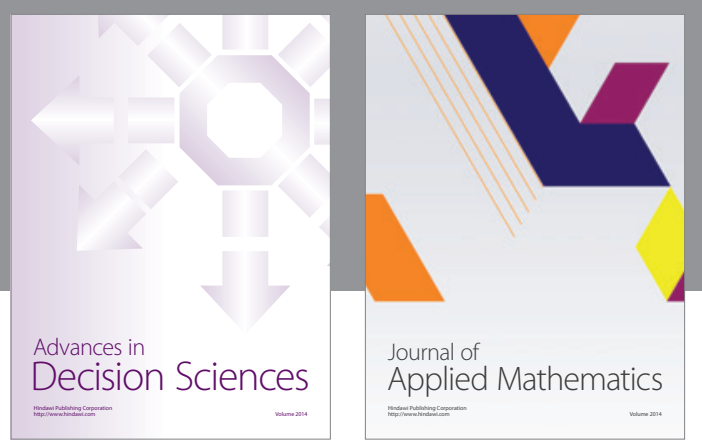

Algebra

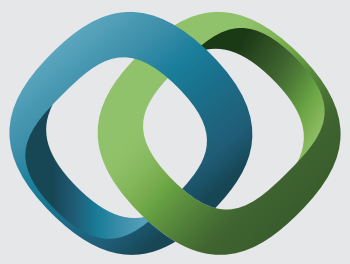

\section{Hindawi}

Submit your manuscripts at

https://www.hindawi.com
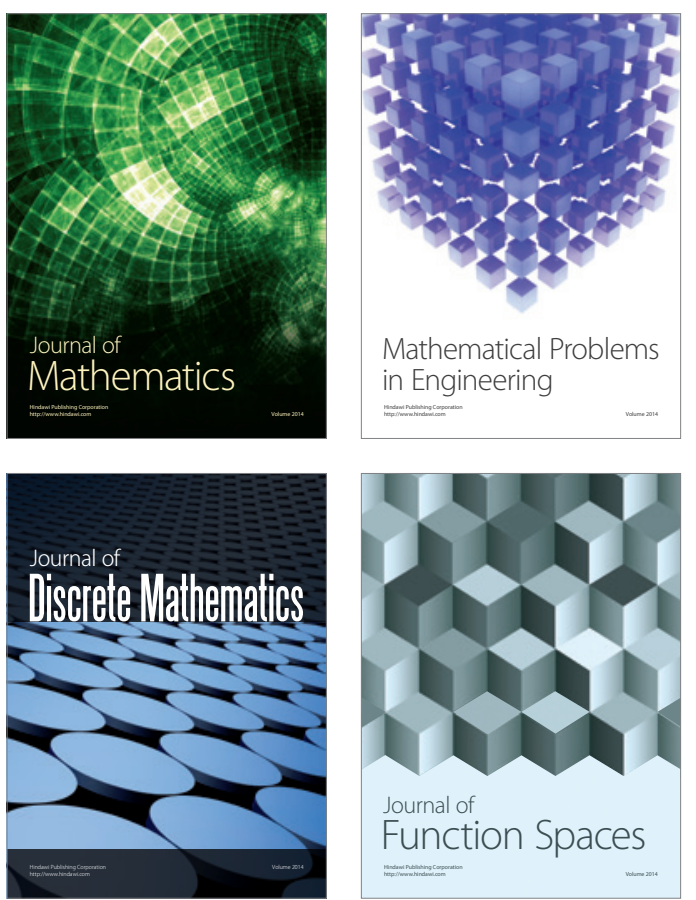

Mathematical Problems in Engineering
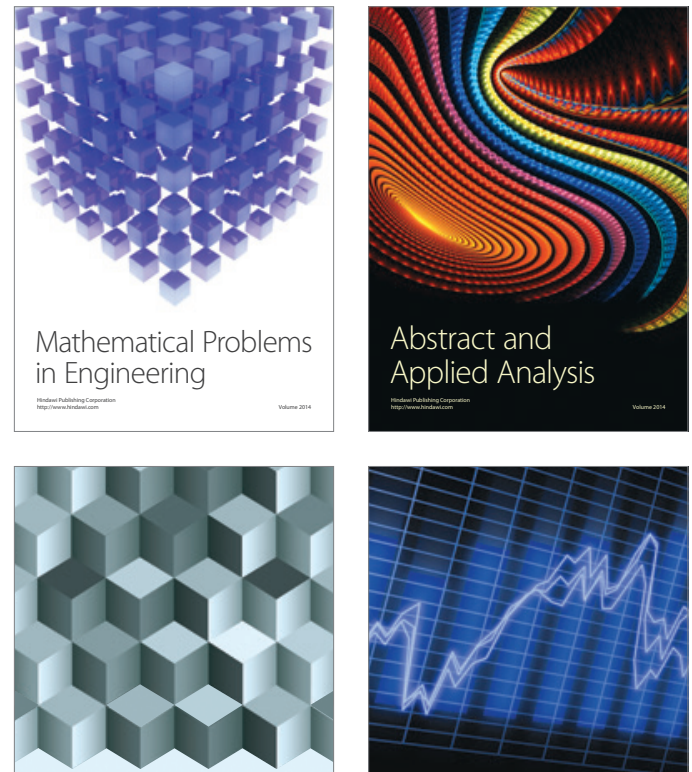

Journal of

Function Spaces

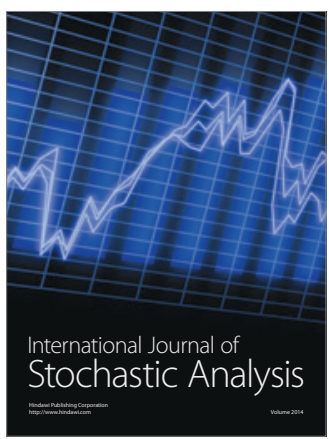

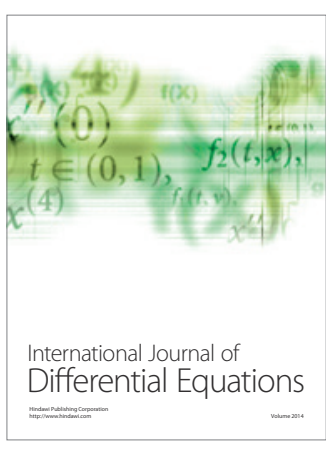
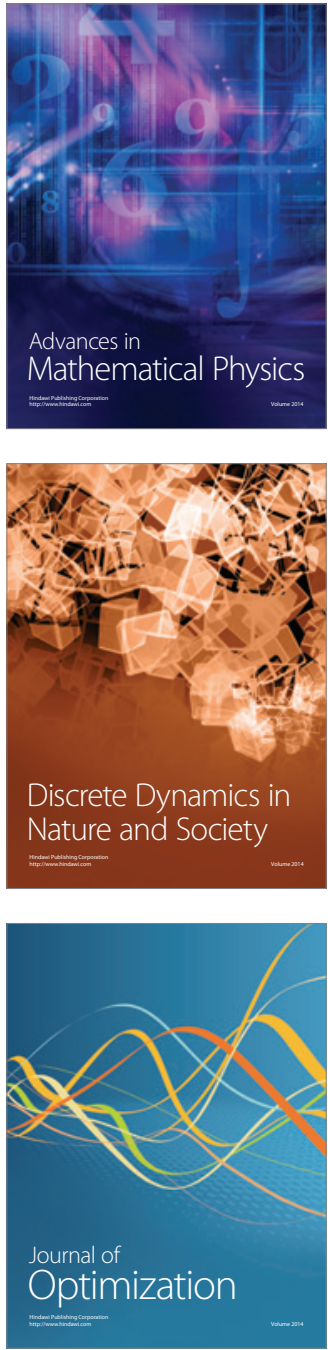